\title{
Sustainability and reducing environmental impacts by recycling rare metals for electric vehicle batteries
}

\author{
T. SAKUNAI ${ }^{1 *}$, L. ITO $^{1}$, A. TOKAI ${ }^{1}$ \\ ${ }^{1}$ Osaka University, Graduate School of Engineering, 2-1 \\ Yamadaoka, Suita, Osaka, 565-0871, Japan \\ ( ${ }^{*}$ correspondence: sakunai@em.see.eng.osaka-u.ac.jp)
}

Currently, lithium-ion batteries are used in a variety of electronic devices, and their demand is expected to increase in the future. Cobalt (Co), nickel (Ni), and lithium (Li), which are used in those batteries, are not only less abundant in the ground, but also require more electrical energy and resources to process ores into the products compared with ordinary metals. Especially, Co is reported to be difficult to secure a stable resource due to the small number of mining countries (METI, 2018), whereas a shortage of high-grade ores causes that of $\mathrm{Ni}$ products, which also increase the environmental impacts such as greenhouse gas (GHG) emissions. However, reuse methods and highly efficient recovery of resources are still under development. Furthermore, Co and Ni should be recovered through the leaching and extraction process by hydrometallurgical treatment to have high purity; however, there are still concerns that these processes require more intensive energy compared to the new mining.

In this study, we investigated the industry sustainability of $\mathrm{Ni}, \mathrm{Co}$, and $\mathrm{Li}$, which are major cathode materials for automotive batteries and are expected to be in short supply. The available amounts from the future generation of waste batteries in Japan were calculated based on the number of years of vehicle use and vehicle penetration rate. We also assumed a closed loop that circulates materials within the same industry in the battery manufacturing industry with the introduction of recycling by hydrometallurgical treatment procedure and evaluated the reduction potential of environmental impacts by recycling in Japan using EverBatt (ALN, 2018), a recycling evaluation model for automotive batteries.

As a result, the incorporation of hydrometallurgical recycling did not change GHG emissions up to the battery manufacturing process, and $\mathrm{Ni}$ was found to be a problem in terms of resource supply and environmental impact that will increase more than three times from the current level in the next ten years despite the recycling due to the rapid increase in demand. Meanwhile, it was found that the supply and mining amounts of Co could be reduced and leveled off after 2025, respectively, by a combination of hydrometallurgical treatment and direct recycle, which can remove only the degraded parts through chemical treatments such as alkaline ones. 